\title{
Cuidado farmacêutico em oncologia: Revisão integrativa da literatura
}

\author{
Pharmaceutical care in oncology: An integrative literature review \\ Atención farmáceutica en oncología: Revision integral de la literatura
}

Recebido: 12/04/2021 | Revisado: 18/04/2021 | Aceito: 25/04/2021 | Publicado: 10/05/2021

\author{
Angela Valéria de Araújo Batista \\ ORCID: https://orcid.org/0000-0002-7566-961X \\ Fundação Santa Casa de Misericórdia do Pará, Brasil \\ E-mail: angelabatista1329@gmail.com \\ Valéria Regina Cavalcante dos Santos \\ ORCID: https://orcid.org/0000-0002-1264-8125 \\ Fundação Santa Casa de Misericórdia do Pará, Brasil \\ E-mail: valregsantos@hotmail.com \\ Irna Carla do Rosário Souza Carneiro \\ ORCID: https://orcid.org/0000-0002-6797-7735 \\ Fundação Santa Casa de Misericórdia do Pará, Brasil \\ E-mail: irnajula@gmail.com
}

\begin{abstract}
Resumo
O câncer se caracteriza como um conjunto de doenças em que as células anormais crescem e disseminam-se descontroladamente. Por isso, é necessária a realização da atenção farmacêutica que se tornou fundamental durante o tratamento oncológico, pois é o farmacêutico o profissional responsável por sanar dúvidas e proporcionar as orientações do uso correto dos medicamentos, assim como acompanhar possíveis reações adversas ou interações medicamentosas. Essa revisão integrativa teve como objetivo avaliar as bibliografias disponíveis sobre o cuidado farmacêutico em oncologia. A pesquisa foi realizada entre os anos de 2016 a 2020 através dos descritores "cuidados farmacêuticos", "atenção farmacêutica", "oncologia", "quimioterapia", "assistência farmacêutica", "paciente oncológico" e "câncer". As bases de dados utilizadas foram: Scientific Electronic Library Online (SciELO), Biblioteca Virtual em Saúde (BVS), Literatura Latino-Americana e do Caribe em Ciências da Saúde (LILACS), no Portal de Periódicos da CAPES e no Google Acadêmico. Os critérios de inclusão utilizados foram artigos completos disponíveis eletronicamente, escritos em português e adequados ao tema escolhido. Depois de utilizados os critérios de inclusão e exclusão citados na metodologia deste trabalho, foram incluídos nessa revisão integrativa da literatura 16 artigos, os quais versam acerca do cuidado farmacêutico em pacientes oncológicos. Destes, 4 foram encontrados no Portal de Periódicos da CAPES, 2 na base de dados da SciELO e 10 no Google Acadêmico; as demais bases pesquisadas não obtiveram resultados incluídos. Apesar de já existirem muitos trabalhos sobre cuidados farmacêuticos na literatura, ainda são poucos os estabelecimentos de saúde que prestam adequadamente esse tipo de serviço ou que o possuem implantado. Portanto a necessidade em trabalhos futuros de verificar as instituições que já possuem seus instrumentos de cuidado farmacêuticos em uso.
\end{abstract}

Palavras-chave: Cuidados farmacêuticos; Atenção farmacêutica; Oncologia; Quimioterapia; Assistência farmacêutica.

\begin{abstract}
Cancer is characterized as a set of diseases in which abnormal cells grow and spread uncontrollably. Therefore, it is necessary to perform the pharmaceutical care that has become essential during cancer treatment, as the pharmacist is the professional responsible for solving doubts and providing guidelines for the correct use of medicines, as well as monitoring possible adverse reactions or drug interactions. This integrative review aimed to evaluate the available bibliographies on pharmaceutical care in oncology. The research was carried out between the years 2016 to 2020 using the keywords "pharmaceutical care", "pharmaceutical care", "oncology", "chemotherapy", "pharmaceutical care", "cancer patient" and "cancer". The databases used were: Scientific Electronic Library Online (SciELO), Virtual Health Library (VHL), Latin American and Caribbean Literature in Health Sciences (LILACS), in the CAPES Journal Portal and in Google Scholar. The inclusion criteria used were complete articles available electronically, written in Portuguese and appropriate to the chosen theme. After using the inclusion and exclusion criteria mentioned in the methodology of this study, 16 articles were included in this integrative literature review, which deal with pharmaceutical care in cancer patients. Of these, 4 were found on the CAPES Journal Portal, 2 on the SciELO database and 10 on Google Scholar; the other bases surveyed did not obtain results included. Although there are already many studies on pharmaceutical care in the literature, there are still few health establishments that adequately provide this type of service or that have it implemented. Therefore, the need for future work to verify the institutions that already have their pharmaceutical care instruments in use.
\end{abstract}


Keywords: Pharmaceutical care; Pharmaceutical attention; Oncology; Chemotherapy; Pharmaceutical assistance.

\begin{abstract}
Resumen
El cáncer se caracteriza por un conjunto de enfermedades en las que las células anormales crecen y se diseminan de manera incontrolable. Por tanto, es necesario realizar los cuidados farmacéuticos que se han convertido en imprescindibles durante el tratamiento del cáncer, ya que el farmacéutico es el profesional responsable de resolver dudas y dar pautas para el correcto uso de los medicamentos, así como de monitorizar las posibles reacciones adversas o interacciones medicamentosas. Esta revisión integradora tuvo como objetivo evaluar las bibliografías disponibles sobre atención farmacéutica en oncología. La investigación se realizó entre los años 2016 a 2020 utilizando las palabras clave "atención farmacéutica", "atención farmacéutica", "oncología", "quimioterapia", "atención farmacéutica", "paciente oncológico" y "cáncer". Las bases de datos utilizadas fueron: Scientific Electronic Library Online (SciELO), Virtual Health Library (BVS), Literatura Latinoamericana y del Caribe en Ciencias de la Salud (LILACS), CAPES Journal Portal y Google Scholar. Los criterios de inclusión utilizados fueron artículos completos disponibles en formato electrónico, escritos en portugués y adecuados al tema elegido. Después de utilizar los criterios de inclusión y exclusión mencionados en la metodología de este estudio, se incluyeron en esta revisión integradora de la literatura 16 artículos que tratan de la atención farmacéutica en pacientes con cáncer. De estos, 4 se encontraron en el CAPES Journal Portal, 2 en la base de datos SciELO y 10 en Google Scholar; las otras bases encuestadas no obtuvieron resultados incluidos. Si bien ya existen muchos estudios sobre la atención farmacéutica en la literatura, aún son pocos los establecimientos de salud que brindan adecuadamente este tipo de servicio o que lo tienen implementado. De ahí la necesidad de un trabajo futuro para verificar las instituciones que ya tienen en uso sus instrumentos de atención farmacéutica.
\end{abstract}

Palabras clave: Cuidado farmacéutico; Atención farmacéutica; Oncología; Quimioterapia; Asistencia farmacéutica.

\title{
1. Introdução
}

O câncer se caracteriza como uma série de doenças em que as células anormais crescem e disseminam-se descontroladamente. O último processo é chamado de metástase caracterizada como principal causa de morte por câncer. A metástase ocorre com a invasão de órgãos e tecidos envolvidos no processo (WHO, 2018).

De acordo com dados do Instituto Nacional do Câncer (INCA, 2019), estima-se que no Brasil para cada ano do triênio 2020-2022 ocorrerão 625 mil casos novos de câncer; 450 mil, excluindo os casos de câncer de pele não melanoma. O câncer de pele não melanoma será o mais incidente (177 mil), seguido pelos cânceres de mama e próstata (66 mil cada), cólon e reto (41 mil), pulmão (30 mil) e estômago (21 mil). O câncer foi a segunda principal causa de morte em todo o mundo, representando uma estimativa de 9,6 milhões de mortes, ou uma em cada seis mortes em 2018 (WHO, 2018).

Com isso, a atenção/ cuidado farmacêutico tornou-se fundamental durante tratamento oncológico, pois o farmacêutico é o profissional responsável por sanar duvidas e proporcionar as orientações sobre a utilização adequada dos medicamentos, assim como acompanhar as reações adversas ou interações medicamentosas, mantendo assim os pacientes amparados quanto às informações relacionadas à ação dos fármacos e ao desenvolvimento da terapia farmacológica, podendo assim contribuir de forma significativa para um bom prognóstico (Rech, Francellino, Colacite. 2019).

No Brasil, a fase de cuidado farmacêutico ainda é insipiente, tendo iniciativas isoladas de alguns farmacêuticos no território nacional nos diversos níveis de atenção à saúde. Os primeiros documentos técnicos que serviram para padronizar e reconhecer a prática do cuidado farmacêutico foram publicados na década de 2000. No entanto, a regulamentação e os documentos que norteiam essa prática, tanto no setor público como no privado, vêm sendo construídos e publicados até os dias atuais (Profarma, 2018).

Para que esses aspectos sejam, de fato, facilitadores, é imprescindível que o farmacêutico atue de forma integrada à equipe de saúde e articulada com a gestão dos serviços, desde a implantação até a mensuração dos resultados. Por outro lado, por se tratar de um novo modelo de prática no contexto da profissão farmacêutica, é natural que desafios sejam frequentes, desde a formação do profissional até a avaliação dos resultados no contexto dos serviços de saúde (Profarma, 2018). 
Segundo um estudo realizado por Silva et al (2017) a atenção farmacêutica é importante nesse processo, orientando o uso correto dos medicamentos, acompanhando reações adversas e interações medicamentosas, diminuindo o risco de erros e a descontinuidade do tratamento.

A atuação do farmacêutico no tratamento oncológico ainda é pequeno, mas vem evoluindo para além da dispensação de medicamentos, pois esse profissional busca encontrar e resolver eventos que possam ter relação a terapia medicamentosa que apareçam durante o tratamento. Portanto, sua inserção na equipe multiprofissional do tratamento oncológico pode contribuir de forma positiva para o alcance da máxima efetividade e segurança da farmacoterapia e melhoria na qualidade de vida e bem-estar do paciente (Rech, Francellino, Colacite. 2019).

A presente investigação é uma revisão integrativa da literatura que teve como objetivo avaliar as evidências disponíveis sobre o cuidado farmacêutico em oncologia, contribuir e somar esforços para a melhoria da assistência farmacêutica em pacientes diagnosticados com neoplasias malignas.

\section{Metodologia}

O presente estudo consiste em uma revisão integrativa de literatura sobre o cuidado farmacêutico e/ou atenção farmacêutica em pacientes oncológicos. Para realizar essa revisão integrativa, as seguintes etapas foram percorridas: definição do tema, elaboração da pergunta norteadora, estabelecimento de critérios de busca ou amostragem na literatura, definição das informações extraídas dos artigos (coleta de dados), análise crítica dos artigos incluídos, discussão e apresentação dos resultados.

Para nortear a revisão integrativa, elaborou-se a seguinte questão: Quais as melhorias ao paciente oncológico com a inserção do cuidado farmacêutico?

A pesquisa foi realizada entre os anos de 2016 a 2020 nas bases de dados descritas abaixo, utilizando os Descritores de Ciências da Saúde (DeCS).

Os descritores selecionados foram "cuidados farmacêuticos", "atenção farmacêutica", "oncologia", "quimioterapia", "assistência farmacêutica", "paciente oncológico" e "câncer"; algumas vezes foi utilizado o operador booleano "AND".

As pesquisas foram realizadas utilizando as bases de dados: SciELO e BVS. Também foram buscados artigos nas bases da LILACS, no Portal de Periódicos da CAPES e no Google Acadêmico.

Utilizou-se como critérios de inclusão artigos com resumo e texto completos disponíveis eletronicamente, escritos em português, publicados de 2016 a 2020 em periódicos científicos e que estivessem adequados ao tema proposto para o trabalho.

Foram excluídos do estudo, artigos que não abordaram, em conjunto, os temas: cuidado farmacêutico e/ou atenção farmacêutica em pacientes oncológicos, artigos que abordaram esses aspectos, porém, em outras doenças, que não o câncer, artigos duplicados nas bases de dados, artigos de revisão que não estavam disponíveis na íntegra ou que não eram artigos científicos e não estavam em português. 
Figura 1 - Esquema da quantidade de artigos utilizados.

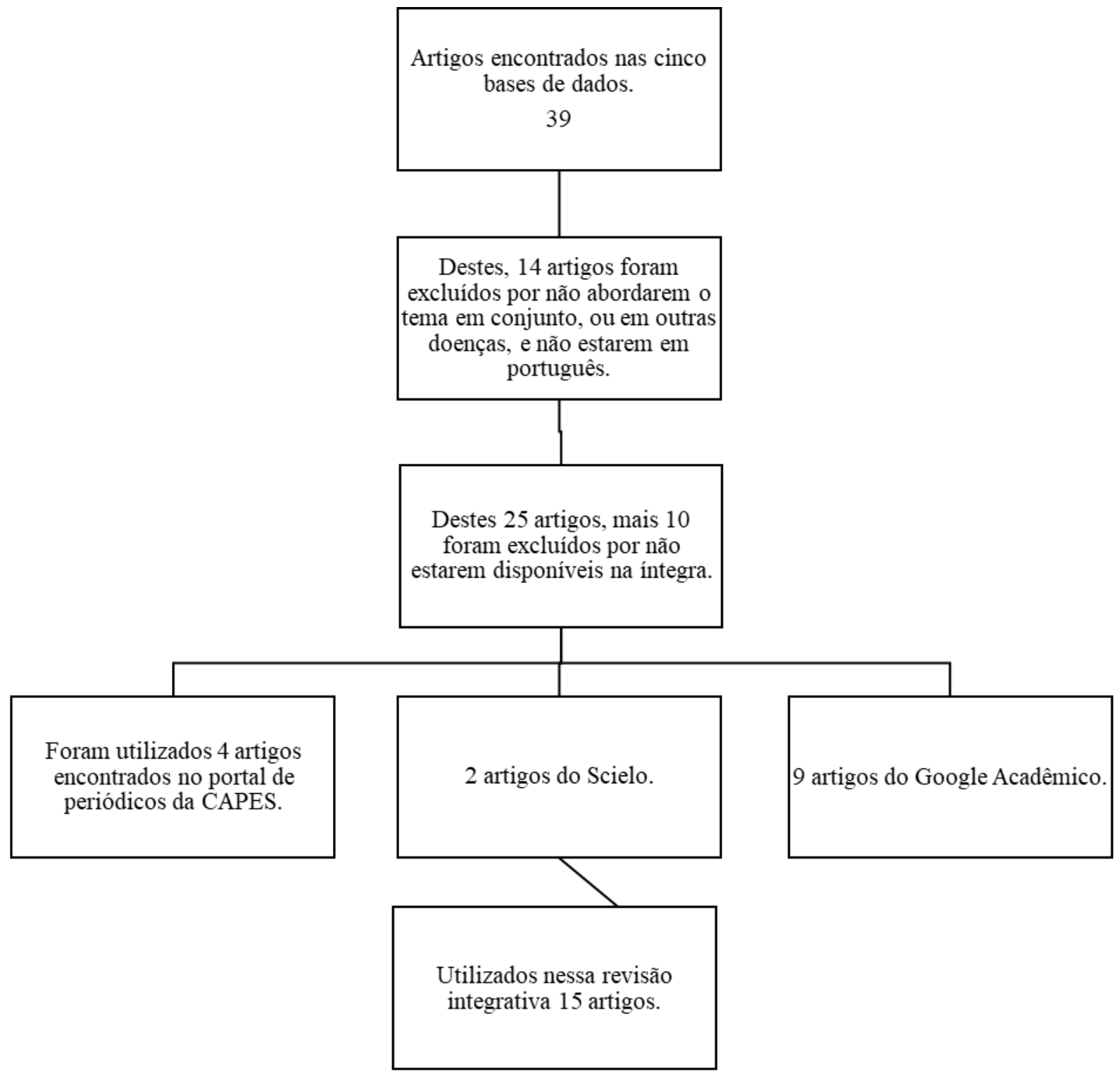

Fonte: Autoras (2021).

\section{Resultados e Discussão}

Depois de utilizados os critérios de inclusão e exclusão citados na metodologia desse trabalho, foram incluídos nessa revisão integrativa da literatura 16 artigos, os quais versam acerca do cuidado farmacêutico em pacientes oncológicos. Destes, 4 foram encontrados no Portal de Periódicos da CAPES, 2 na base de dados da SciELO e 10 no Google Acadêmico; as demais bases pesquisadas não obtiveram resultados incluídos.

$\mathrm{Na}$ referida revisão integrativa, analisou-se 16 artigos que estão apresentados no quadro 1 de forma sucinta, possibilitando uma visão geral dos artigos que foram incluídos.

Os artigos relatam sobre a importância do cuidado farmacêutico para o tratamento do paciente oncológico, objetivando com isso uma melhora em seu caso clínico e na qualidade de vida durante o tratamento. Além de ajudar no manejo de reações adversas e mostrar propostas de estruturação na assistência farmacêutica prestada a esses pacientes. 
O resultado da pesquisa demostrou a necessidade do serviço de implantação e estruturação de instrumentos para realização de consultas de cuidado farmacêutico, assim como mostrou em um estudo realizado na Unidade Básica de Saúde (UBS) de Curitiba (PR), com o objetivo de analisar os resultados da implantação de um serviço de clínica farmacêutica, observou-se o perfil nosológico de 876 pacientes atendidos na atenção primária do município, que indicou alta prevalência de condições crônicas na população idosa, bem como, problemas relacionados à farmacoterapia em grande parte dos pacientes. $\mathrm{Na}$ realização das intervenções farmacêuticas para reverter o quadro dos problemas, foi observada a dificuldade em ter apenas uma consulta para intervir de forma segura na medicação dos pacientes, sendo necessário mais tempo de acompanhamento (Lombardi, 2016).

De acordo com o estudo de Chan et al (2013), a intervenção farmacêutica tem um alto impacto e é desejável para o tratamento, otimizando os custos e garantindo uma prescrição mais segura, reafirmando a importância dos serviços farmacêuticos.

O acompanhamento do farmacêutico aos pacientes em tratamento oncológico é uma importante ferramenta para a redução de erros durante o tratamento, tornando-o mais eficaz e melhorando a qualidade da assistência. A tarefa desse profissional é garantir que a terapia medicamentosa esteja devidamente adequada e que seja a mais segura e conveniente ao paciente. Neste sentido a Atenção Farmacêutica pode ter um papel importante neste processo (Leão. et al. 2012).

A atuação do farmacêutico clínico, através do trabalho de atenção farmacêutica, junto à equipe multiprofissional, visa promover a qualidade terapêutica do paciente, uma vez que orienta os profissionais sobre o uso seguro e racional dos medicamentos. Este profissional é importante na identificação, correção e redução de possíveis riscos associados à terapia, sendo o paciente o principal beneficiário das suas ações (Ribeiro. et al. 2009).

Compete ao farmacêutico acompanhar diariamente o trabalho da equipe buscando agregar seus conhecimentos farmacológicos na qualidade do trabalho assistencial (Ferracini, Filho. 2012).

A Farmácia Clínica pressupõe que o farmacêutico estabeleça um relacionamento ativo com os demais membros da equipe de saúde, principalmente médicos e enfermeiros. Porém, também pressupõe o contato com os pacientes, para assim assegurar resultados clinicamente apropriados para a farmacoterapia, o paciente é o objeto principal das atividades do farmacêutico hospitalar (Storpitis. 2008). 
Quadro 1 - Resumo dos artigos levantados nas bases de dados pesquisadas sobre revisão integrativa.

\begin{tabular}{|c|c|c|c|c|c|}
\hline Autores & Título & Local de Busca & Tipo de Pesquisa & Descritores & $\begin{array}{c}\text { Ano de } \\
\text { Publicação }\end{array}$ \\
\hline $\begin{array}{l}\text { 1-Juliana dos Santos Silva; } \\
\text { Fyrino Rolipe } \\
\text { Braga. }\end{array}$ & $\begin{array}{l}\text { Cuidado Farmacêutico a } \\
\text { pacientes em tratamento com } \\
\text { Herceptin } \AA \text { (Transtuzumabe): um } \\
\text { estudo de caso. }\end{array}$ & $\begin{array}{l}\text { Portal de Periódicos da } \\
\text { CAPES. }\end{array}$ & Estudo de Caso. & $\begin{array}{l}\text { Cuidado farmacêutico; } \\
\text { Educação em saúde; Adesão à farmacoteraplásicos; }\end{array}$ & 2019 \\
\hline $\begin{array}{l}\text { 2-Jacqueline Aragão de Medeiros; } \\
\text { Aline Patrícia Fonseca Macêdo de } \\
\text { Melo; Vivian Mariano Torres. }\end{array}$ & $\begin{array}{l}\text { Atuação do farmacêutico clínico } \\
\text { hospitalar em pacientes } \\
\text { oncológicos frente ao avanço na } \\
\text { legislação brasileira. }\end{array}$ & $\begin{array}{l}\text { Portal de Periódicos da } \\
\text { CAPES. }\end{array}$ & Revisão Integrativa. & $\begin{array}{l}\text { Assistência } \quad \text { Farmacêutica; } \quad \text { Oncologia; } \\
\text { Antineoplásicos; Legislação Farmacêutica. }\end{array}$ & 2019 \\
\hline $\begin{array}{l}\text { 3-Caroline Oliveira Rangel; Gabriela } \\
\text { Tassotti Gelatti; Rodrigo Fernando dos } \\
\text { Santos Salazar; Christiane de Fátima } \\
\text { Colet ; Vanessa Adelina Casali } \\
\text { Bandeira; Roberta Cattaneo Horn. }\end{array}$ & $\begin{array}{l}\text { Avaliação da adesão ao } \\
\text { tratamento com Tamoxifeno por } \\
\text { mulheres com câncer de mama. }\end{array}$ & $\begin{array}{l}\text { Portal de Periódicos da } \\
\text { CAPES. }\end{array}$ & $\begin{array}{l}\text { Estudo Clínico } \\
\text { Randomizado. }\end{array}$ & $\begin{array}{l}\text { Adesão à medicação; Antineoplásicos } \\
\text { hormonais; Atenção Farmacêutica; Neoplasias } \\
\text { de Mama; Oncologia. }\end{array}$ & 2020 \\
\hline $\begin{array}{l}\text { 4-Deysiane Dos Santos Calado; Diego } \\
\text { de Hollanda Cavalcanti Tavares; } \\
\text { Grasiela Costa Bezerra. }\end{array}$ & $\begin{array}{l}\text { O papel da atenção farmacêutica } \\
\text { na redução das reações adversas } \\
\text { associados ao tratamento de } \\
\text { pacientes oncológicos. }\end{array}$ & $\begin{array}{l}\text { Portal de Periódicos da } \\
\text { CAPES. }\end{array}$ & Revisão Bibliográfica. & $\begin{array}{l}\text { Câncer; Farmacoterapia; Paciente Oncológico; } \\
\text { Farmacêutico Clínico. }\end{array}$ & 2019 \\
\hline $\begin{array}{l}\text { 5-Mario Jorge Sobreira da Silva; } \\
\text { Claudia Garcia Serpa Osorio-de-Castro. }\end{array}$ & $\begin{array}{l}\text { Organização e práticas da } \\
\text { assistência farmacêutica em } \\
\text { oncologia no âmbito do Sistema } \\
\text { Único de Saúde. }\end{array}$ & SciELO. & Estudo de caso. & $\begin{array}{l}\text { Assistência Farmacêutica; Oncologia; Estudo } \\
\text { de casos; Assistência à saúde; Sistema Único } \\
\text { de Saúde. }\end{array}$ & 2019 \\
\hline $\begin{array}{l}\text { 6-Sandna Larissa Freitas dos Santos, } \\
\text { Hérick Hebert da Silva Alves, Cinara } \\
\text { Vidal Pessoa, Hévila Suellen Teixeira } \\
\text { Tavares Saraiva, Karla Bruna Nogueira } \\
\text { Torres Barros. }\end{array}$ & $\begin{array}{l}\text { Evidências do cuidado } \\
\text { farmacêutico na prática } \text { clínica } \\
\text { da oncologia. }\end{array}$ & Google acadêmico. & Revisão de literatura. & $\begin{array}{l}\text { Assistência farmacêutica; conduta do } \\
\text { tratamento medicamentoso; antineoplásicos; } \\
\text { farmacêuticos; oncologia; qualidade de vida. }\end{array}$ & 2018 \\
\hline $\begin{array}{l}\text { 7-Juliana Pereira dos Santos; Regina } \\
\text { Maria da Hora dos Santos Azevedo; } \\
\text { Patrícia Lima de Araújo; Maria Teresita } \\
\text { Bendicho; Rosa Malena Fagundes } \\
\text { Xavier. }\end{array}$ & $\begin{array}{l}\text { Cuidado farmacêutico em UTI } \\
\text { oncológica. }\end{array}$ & Google acadêmico. & Relato de experiência. & $\begin{array}{l}\text { Oncologia; } \quad \text { Cuidado } \\
\text { Intervenções farmacêuticas. }\end{array}$ & 2020 \\
\hline
\end{tabular}




\begin{tabular}{|c|c|c|c|c|c|}
\hline $\begin{array}{l}\text { 8-Rainne de Oliveira Almeida; Dayse } \\
\text { Aparecida Oliveira Braga; Maria Alinne } \\
\text { de Souza; Karla Bruna Nogueira Torres } \\
\text { Barros; Leina Mércia de Oliveira } \\
\text { Vasconcelos. }\end{array}$ & $\begin{array}{l}\text { Implantação do cuidado } \\
\text { farmacêutico em pacientes } \\
\text { Oncológicos em um núcleo de } \\
\text { apoio à saúde da família. }\end{array}$ & Google acadêmico. & Estudo descritivo. & $\begin{array}{l}\text { Atenção } \quad \text { Farmacêutica; } \quad \text { Oncologia; } \\
\text { Farmacoterapia. }\end{array}$ & 2017 \\
\hline $\begin{array}{l}\text { 9-Fernanda Fávero Alberti; Manuela } \\
\text { Borges Sangoi Cardoso; Liana Pedrolo } \\
\text { Canterle; Eduardo Kraetzig Donini. }\end{array}$ & $\begin{array}{l}\text { Cuidado farmacêutico aplicado a } \\
\text { mulheres com câncer de mama na } \\
\text { Atenção Primária à Saúde. }\end{array}$ & Google acadêmico. & $\begin{array}{l}\text { Estudo longitudinal } \\
\text { prospectivo. }\end{array}$ & $\begin{array}{l}\text { Acompanhamento farmacoterapêutico; } \\
\text { Farmácia Clínica; Pacientes oncológicos. }\end{array}$ & 2018 \\
\hline $\begin{array}{l}\text { 10-Michelly Venceslau Vendramini } \\
\text { Simões; Jefferson Silva Martins; Silvia } \\
\text { de Lima Vieira; Wanessa Cassemiro } \\
\text { Fernandes; Claudinei Alves Santana. }\end{array}$ & $\begin{array}{l}\text { Cuidados farmacêuticos na } \\
\text { adesão da terapia medicamentosa } \\
\text { oral em pacientes onco- } \\
\text { pediátricos. }\end{array}$ & Google acadêmico. & Revisão de literatura. & $\begin{array}{l}\text { Adesão à medicação oral; Câncer pediátrico; } \\
\text { orientação farmacêutica. }\end{array}$ & 2020 \\
\hline $\begin{array}{l}\text { 11-Erica Assis Alves, Gabriel } \\
\text { Guimarães Tavares1, Leonardo Luiz } \\
\text { Borges. }\end{array}$ & $\begin{array}{lrr}\text { Importância da atenção } \\
\text { farmacêutica para } \quad a \\
\text { quimioterapia } & \text { antitumoral. }\end{array}$ & Google acadêmico. & Revisão de literatura. & Atenção Farmacêutica; Câncer; Oncologia. & 2020 \\
\hline $\begin{array}{l}\text { 12-Karina da Silva Aguiar; Jamile } \\
\text { Machado dos Santos; Mônica Cristina } \\
\text { Cambrussi; Solane Picolotto; Marcela } \\
\text { Bechara Carneiro. }\end{array}$ & $\begin{array}{l}\text { Segurança do paciente e o valor } \\
\text { da intervenção farmacêutica em } \\
\text { um hospital oncológico. }\end{array}$ & SciELO. & Estudo observacional. & $\begin{array}{l}\text { Farmacoeconomia; Assistência farmacêutica; } \\
\text { Erros de medicação; Prescrições de } \\
\text { medicamentos; Antineoplásicos; Serviço } \\
\text { hospitalar de oncologia. }\end{array}$ & 2017 \\
\hline $\begin{array}{l}\text { 13-Laís de Souza Gonçalves Eugênio; } \\
\text { Osni Lázaro Pinheiro. }\end{array}$ & $\begin{array}{l}\text { Paciente oncológico em } \\
\text { tratamento medicamentoso: } \\
\text { Subsídios para implantação de } \\
\text { um programa de atenção } \\
\text { Farmacêutica. }\end{array}$ & Google acadêmico. & Estudo observacional. & $\begin{array}{l}\text { Atenção farmacêutica; Pacientes ambulatoriais; } \\
\text { Oncologia; } \\
\text { Administração oral. }\end{array}$ & 2018 \\
\hline $\begin{array}{l}\text { 14-Priscila Helena Marietto Figueira; } \\
\text { Renata Rosa Veloso Cataldo; Elaine } \\
\text { Silva Miranda; Gabriela Bittencourt } \\
\text { Gonza-lez Mosegui. }\end{array}$ & 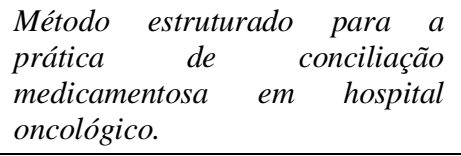 & Google acadêmico. & $\begin{array}{l}\text { Estudo retrospectivo, } \\
\text { exploratório } \\
\text { descritivo. }\end{array}$ & $\begin{array}{l}\text { Gestão da Terapia Medicamentosa, Segurança } \\
\text { do Paciente, Gestão de risco, Erros de } \\
\text { Medicação. }\end{array}$ & 2019 \\
\hline $\begin{array}{l}\text { 15-Lívia Christina Almeida da Silva; } \\
\text { Poliana Oliveira Lemos de Brito; } \\
\text { Cristiane Dominice Melo; Angela } \\
\text { Falcai; Ione Cristina de Paiva Pereira. }\end{array}$ & $\begin{array}{l}\text { Contribuições da atenção } \\
\text { farmacêutica a pacientes em } \\
\text { tratamento Oncológico. }\end{array}$ & Google acadêmico. & Revisão de literatura. & $\begin{array}{l}\text { Atenção Farmacêutica; } \quad \text { Farmacoterapia; } \\
\text { Paciente oncológico. }\end{array}$ & 2017 \\
\hline
\end{tabular}

Fonte: Autoras (2021). 
Quadro 2 - Apresentação da síntese dos artigos da Revisão Integrativa.

\begin{tabular}{|c|c|c|c|c|}
\hline Autores & Título da Pesquisa & Detalhamento Metodológico & Resultados Principais & Conclusões/Recomendações \\
\hline $\begin{array}{l}\text { 1-Juliana dos Santos Silva; Felipe } \\
\text { Cyrino Rodrigues; Raquel Rennó } \\
\text { Braga. }\end{array}$ & $\begin{array}{l}\text { Cuidado Farmacêutico a } \\
\text { pacientes em tratamento com } \\
\text { Herceptin }{ }^{\circledR} \\
\text { (Transtuzumabe): um estudo } \\
\text { de caso. }\end{array}$ & $\begin{array}{l}\text { O atendimento farmacêutico foi } \\
\text { realizado com quatro pacientes } \\
\text { mulheres com câncer de mama } \\
\text { metastático que superexpressam HER-2 } \\
\text { e que realizavam o tratamento com o } \\
\text { antineoplásico Herceptin }{ }^{\circledR} \\
\text { (Trastuzumabe), por um período de } 4 \\
\text { (quatro) meses no ano de } 2016 .\end{array}$ & $\begin{array}{l}\text { Os principais Problemas Relacionados a } \\
\text { Medicamentos (PRM) identificados durante } \\
\text { os atendimentos foram de segurança, } \\
\text { relacionados a reações adversas do } \\
\text { tratamento. Foram realizadas intervenções } \\
\text { farmacêuticas junto às pacientes e aos } \\
\text { prescritores. }\end{array}$ & $\begin{array}{l}\text { As intervenções educativas ajudaram a } \\
\text { melhorar a compreensão das pacientes } \\
\text { sobre a doença e o tratamento, contribuindo } \\
\text { para a manutenção da farmacoterapia. O } \\
\text { presente trabalho evidencia a importância } \\
\text { da atuação do farmacêutico na melhora da } \\
\text { qualidade de vida das pacientes } \\
\text { acompanhadas. }\end{array}$ \\
\hline $\begin{array}{l}\text { 2-Jacqueline Aragão de Medeiros; } \\
\text { Aline Patrícia Fonseca Macêdo de } \\
\text { Melo; Vivian Mariano Torres. }\end{array}$ & $\begin{array}{l}\text { Atuação do farmacêutico } \\
\text { clínico hospitalar em } \\
\text { pacientes oncológicos frente } \\
\text { ao avanço na legislação } \\
\text { brasileira. }\end{array}$ & $\begin{array}{l}\text { Foi realizada uma revisão integrativa } \\
\text { cujos resultados foram obtidos por meio } \\
\text { de uma pesquisa exploratória. Foram } \\
\text { selecionados } 17 \text { artigos no período de } \\
2008 \text { a 2018, referentes ao tema } \\
\text { proposto. }\end{array}$ & $\begin{array}{l}\text { Com os artigos comprovou-se que a } \\
\text { assistência farmacêutica gera resultados } \\
\text { positivos na terapia, evitando erros de } \\
\text { prescrição e problemas relacionados a } \\
\text { medicamentos, assim como diminui custos } \\
\text { para os hospitais. }\end{array}$ & $\begin{array}{l}\text { Conforme a exposição dos dados, conclui- } \\
\text { se a importância da assistência farmacêutica } \\
\text { no tratamento oncológico, possibilitando } \\
\text { uma terapêutica segura e eficaz. }\end{array}$ \\
\hline $\begin{array}{l}\text { 3-Caroline Oliveira Rangel; } \\
\text { Gabriela Tassotti Gelatti; Rodrigo } \\
\text { Fernando dos Santos Salazar; } \\
\text { Christiane de Fátima Colet; } \\
\text { Vanessa Adelina Casali Bandeira; } \\
\text { Roberta Cattaneo Horn. }\end{array}$ & $\begin{array}{l}\text { Avaliação da adesão ao } \\
\text { tratamento com Tamoxifeno } \\
\text { por mulheres com câncer de } \\
\text { mama. }\end{array}$ & $\begin{array}{l}\text { Trata-se de um estudo clínico } \\
\text { randomizado e quantitativo. A coleta de } \\
\text { dados foi realizada durante seis meses. } \\
\text { A randomização aleatória dividiu-se em } \\
\text { Grupo Controle e Grupo } \\
\text { Acompanhamento, sendo o } \\
\text { acompanhamento subdividido: Antes e } \\
\text { Após o Acompanhamento. O Grupo } \\
\text { acompanhamento recebeu mensalmente } \\
\text { intervenções farmacêuticas individuais. }\end{array}$ & $\begin{array}{l}\text { Após acompanhamento farmacêutico } \\
\text { observou-se diferença entre os grupos Grupo } \\
\text { Acompanhamento e Grupo Controle quanto à } \\
\text { prática de atividade física }(\mathrm{p}=0,043) \text {, adesão } \\
\text { ao tratamento }(\mathrm{p}=0,006) \text {, redução de efeitos } \\
\text { adversos ( } \mathrm{p}=0,003) \text { e doenças associadas } \\
(\mathrm{p}=0,002) \text {. Os PRMs mais frequentes foram } \\
\text { de segurança e adesão, para os quais foram } \\
\text { realizadas } 54 \text { intervenções farmacêuticas. }\end{array}$ & $\begin{array}{l}\text { Evidenciou-se que o acompanhamento } \\
\text { farmacoterapêutico contribuiu efetivamente } \\
\text { na adesão ao tratamento com tamoxifeno e } \\
\text { as intervenções farmacêuticas realizadas } \\
\text { contribuíram para prevenção e redução dos } \\
\text { problemas associados a farmacoterapia. }\end{array}$ \\
\hline $\begin{array}{l}\text { 4-Deysiane Dos Santos Calado; } \\
\text { Diego de Hollanda Cavalcanti } \\
\text { Tavares; Grasiela Costa Bezerra. }\end{array}$ & $\begin{array}{l}\text { O papel da atenção } \\
\text { farmacêutica na redução das } \\
\text { reações adversas associados } \\
\text { ao tratamento de pacientes } \\
\text { oncológicos. }\end{array}$ & $\begin{array}{l}\text { Tal pesquisa refere-se a um estudo } \\
\text { descritivo do tipo revisão bibliográfica } \\
\text { narrativa, a coleta de dados foi } \\
\text { realizada nos meses de outubro e } \\
\text { novembro de } 2018 \text { utilizando os } \\
\text { descritores "câncer" e "assistência } \\
\text { farmacêutica". Os critérios de inclusão }\end{array}$ & $\begin{array}{l}\text { Por fim, esse levantamento bibliográfico } \\
\text { também possibilitou mostrar a competência } \\
\text { atribuída ao profissional farmacêutico, que } \\
\text { por muitas vezes é atribuída a outros } \\
\text { profissionais de saúde por vários motivos, } \\
\text { entre eles, destacamos a escassez do } \\
\text { profissional farmacêutico especialista na }\end{array}$ & $\begin{array}{l}\text { O embasamento teórico descrito procurou } \\
\text { apresentar a importância do profissional } \\
\text { farmacêutico na equipe multidisciplinar, no } \\
\text { que diz respeito a suas atribuições e no } \\
\text { desempenho de suas condutas assistenciais } \\
\text { no setor oncológico. Enfatizando a } \\
\text { importância desse profissional quanto aos }\end{array}$ \\
\hline
\end{tabular}




\begin{tabular}{|c|c|c|c|c|}
\hline & & $\begin{array}{l}\text { foram artigos que abordassem o tema } \\
\text { desse estudo e seus descritores, restritos } \\
\text { ao período de } 2008 \text { a } 2018 \text {, no idioma } \\
\text { português, que estivessem disponíveis } \\
\text { na íntegra. }\end{array}$ & clínica oncológica. & $\begin{array}{l}\text { cuidados necessários para redução de } \\
\text { possíveis reações adversas que venham a } \\
\text { ser desenvolvidas, sabendo que o } \\
\text { farmacêutico além da dispensação, tem a } \\
\text { responsabilidade de orientar e acompanhar } \\
\text { a terapia medicamentosa, podendo assim } \\
\text { contribuir significativamente para o êxito } \\
\text { do tratamento. }\end{array}$ \\
\hline $\begin{array}{l}\text { 5-Mario Jorge Sobreira da Silva; } \\
\text { Claudia Garcia Serpa Osorio-de- } \\
\text { Castro. }\end{array}$ & $\begin{array}{l}\text { Organização e práticas da } \\
\text { assistência farmacêutica em } \\
\text { oncologia no âmbito do } \\
\text { Sistema Único de Saúde. }\end{array}$ & $\begin{array}{l}\text { Foram entrevistados } 10 \text { gestores e } 15 \\
\text { profissionais de } \\
\text { Complementarmente, saúde. } \\
\text { considerados documentos e dados da } \\
\text { observação direta da ação dos } \\
\text { profissionais. }\end{array}$ & $\begin{array}{l}\text { Os resultados foram analisados segundo } \\
\text { quatro eixos: estrutura organizacional, } \\
\text { financiamento, tecnologias e processos de } \\
\text { trabalho. Destacaram-se: a baixa articulação } \\
\text { das ações de assistência farmacêutica } \\
\text { realizadas entre os níveis de atenção à saúde, } \\
\text { problemas estruturais nos serviços, } \\
\text { insuficiência de financiamento, atrasos nos } \\
\text { processos de avaliação e incorporação de } \\
\text { tecnologias, e falhas nos processos de } \\
\text { trabalho. }\end{array}$ & $\begin{array}{l}\text { Os aspectos destacados contribuem para a } \\
\text { precariedade do funcionamento do sistema. }\end{array}$ \\
\hline $\begin{array}{l}\text { 6-Sandna Larissa Freitas dos } \\
\text { Santos, Hérick Hebert da Silva } \\
\text { Alves, Cinara Vidal Pessoa, } \\
\text { Hévila Suellen Teixeira Tavares } \\
\text { Saraiva, Karla Bruna Nogueira } \\
\text { Torres Barros. }\end{array}$ & $\begin{array}{l}\text { Evidências do cuidado } \\
\text { farmacêutico na prática } \\
\text { clínica da oncologia. }\end{array}$ & $\begin{array}{l}\text { Trata-se de uma revisão de literatura, } \\
\text { com busca nas bases de dados. Foram } \\
\text { incluídos estudos sobre a temática, } \\
\text { publicados em inglês, português ou } \\
\text { espanhol, em formato de artigos, } \\
\text { revisões, dissertações e teses publicados } \\
\text { entre } 2009 \text { e } 2015 \text {. Foram excluídas as } \\
\text { publicações de anos anteriores e com } \\
\text { duplicidade. }\end{array}$ & $\begin{array}{l}\text { Foi observado que o cuidado farmacêutico } \\
\text { está imerso na terapia medicamentosa, em } \\
\text { tomadas de decisões sobre o uso adequado de } \\
\text { medicamentos para cada paciente oncológico. } \\
\text { Além disso, a realização de orientações e } \\
\text { supervisões nos procedimentos de } \\
\text { manipulação dos antineoplásicos e a atuação } \\
\text { da equipe multiprofissional acompanhando } \\
\text { diariamente o trabalho feito e buscando } \\
\text { agregar seus conhecimentos farmacológicos } \\
\text { são atividades essenciais nesse âmbito. Na } \\
\text { farmacovigilância, o farmacêutico previne as } \\
\text { reações adversas a medicamentos, dada a alta } \\
\text { ocorrência em pacientes sob terapia } \\
\text { quimioterápica. }\end{array}$ & $\begin{array}{l}\text { Dessa forma, o cuidado farmacêutico } \\
\text { garante que o tratamento antineoplásico } \\
\text { seja desenvolvido com qualidade e } \\
\text { segurança, evidenciando a qualidade de } \\
\text { vida do paciente oncológico. }\end{array}$ \\
\hline
\end{tabular}




\begin{tabular}{|c|c|c|c|c|}
\hline $\begin{array}{l}\text { 7-Juliana Pereira dos Santos; } \\
\text { Regina Maria da Hora dos Santos } \\
\text { Azevedo; Patrícia Lima de Araújo; } \\
\text { Maria Teresita Bendicho; Rosa } \\
\text { Malena Fagundes Xavier. }\end{array}$ & $\begin{array}{l}\text { Cuidado farmacêutico em } \\
\text { UTI oncológica. }\end{array}$ & $\begin{array}{l}\text { Estudo descritivo mediante o relato de } \\
\text { experiência de farmacêuticos do } \\
\text { programa de Residência } \\
\text { Multiprofissional em Oncologia da } \\
\text { Universidade do Estado da Bahia } \\
\text { (UNEB), inserido em um hospital } \\
\text { especializado em oncologia, localizado } \\
\text { na capital do estado da Bahia, no } \\
\text { período de março a agosto de } 2019 . \\
\text { Dentre as atividades clínicas, as } \\
\text { variáveis destacadas no trabalho foram } \\
\text { as intervenções farmacêuticas } \\
\text { realizadas e aceitabilidade destas } \\
\text { perante a equipe médica. }\end{array}$ & $\begin{array}{l}\text { Durante o período de março até agosto de } \\
2019,132 \text { pacientes foram acompanhados } \\
\text { pela farmácia clínica e registrados um total de } \\
70 \text { intervenções farmacêuticas, } 34 \% \text { (24) } \\
\text { foram relacionados à introdução de } \\
\text { medicamentos necessários, } 17 \% \text { (12) em } \\
\text { relação ao ajuste da sobredose prescrita, } 16 \% \\
\text { (11) foi de ajuste da subdose prescrita, } 16 \% \\
\text { (11) relacionados ao ajuste da via de } \\
\text { administração, } 9 \% \text { (6) foi suspensão do } \\
\text { medicamento inseguro, } 6 \%(4) \text { das } \\
\text { intervenções foram relacionadas à adesão e } \\
2 \% \text { (2) substituição de medicamento } \\
\text { inseguro. }\end{array}$ & $\begin{array}{l}\text { O acompanhamento fármaco-terapêutico } \\
\text { em UTI oncológica contribui para a } \\
\text { racionalização da terapia farmacológica e } \\
\text { auxilia no cumprimento do plano } \\
\text { terapêutico. }\end{array}$ \\
\hline $\begin{array}{l}\text { 8-Rainne de Oliveira Almeida; } \\
\text { Dayse Aparecida Oliveira Braga; } \\
\text { Maria Alinne de Souza; Karla } \\
\text { Bruna Nogueira Torres Barros; } \\
\text { Leina Mércia de Oliveira } \\
\text { Vasconcelos. }\end{array}$ & $\begin{array}{l}\text { Implantação do cuidado } \\
\text { farmacêutico em pacientes } \\
\text { Oncológicos em um núcleo } \\
\text { de apoio à saúde da família. }\end{array}$ & $\begin{array}{l}\text { Tratando-se de um estudo do tipo } \\
\text { descritivo, observacional, prospectivo, } \\
\text { transversal, com uma abordagem quali- } \\
\text { quantitativa. Foi realizado nas UBSs da } \\
\text { sede do município, sendo os dados } \\
\text { coletados durante os meses de março a } \\
\text { junho de } 2017 \text {. }\end{array}$ & $\begin{array}{l}\text { Dessa forma, o intuito maior deste estudo é } \\
\text { garantir à continuidade do tratamento, } \\
\text { contribuindo diretamente para o sucesso da } \\
\text { terapia, prevenindo e detectando possíveis } \\
\text { Reações Adversas a Medicamentos (RAMs), } \\
\text { efeitos colaterais e eventuais PRMs. }\end{array}$ & $\begin{array}{l}\text { Diante da incidência de casos de câncer e } \\
\text { das diversas reações que a terapia oral pode } \\
\text { ocasionar, faz-se necessário o } \\
\text { acompanhamento por um dos profissionais } \\
\text { mais habilitados para orientar sobre a } \\
\text { tomada da terapia prescrita. }\end{array}$ \\
\hline $\begin{array}{l}\text { 9-Fernanda Fávero Alberti; } \\
\text { Manuela Borges Sangoi Cardoso; } \\
\text { Liana Pedrolo Canterle; Eduardo } \\
\text { Kraetzig Donini. }\end{array}$ & $\begin{array}{l}\text { Cuidado farmacêutico } \\
\text { aplicado a mulheres com } \\
\text { câncer de mama na Atenção } \\
\text { Primária à Saúde. }\end{array}$ & $\begin{array}{l}\text { A metodologia de acompanhamento foi } \\
\text { realizada conforme o Método Dáder. }\end{array}$ & $\begin{array}{l}\text { Foram realizadas } 18 \text { intervenções não- } \\
\text { farmacológicas para as pacientes do estudo, } \\
\text { após serem submetidas há uma média de três } \\
\text { consultas farmacêuticas com cada paciente. A } \\
\text { média de idade das participantes da pesquisa } \\
\text { foi de } 54,1( \pm 8,2) \text { anos. A média de } \\
\text { menopausa das pacientes era de } 44,4( \pm 8,2) \\
\text { anos e a idade de menarca de } 13,2( \pm 2,0) \\
\text { anos. }\end{array}$ & $\begin{array}{l}\text { Verificou-se o quão importante é instituir } \\
\text { na prática farmacêutica as ferramentas de } \\
\text { cuidado e acompanhamento } \\
\text { farmacoterapêutico em diferentes cenários } \\
\text { clínicos. }\end{array}$ \\
\hline
\end{tabular}




\begin{tabular}{|c|c|c|c|c|}
\hline $\begin{array}{lr}\text { 10-Michelly } & \text { Venceslau } \\
\text { Vendramini Simões; Jefferson } \\
\text { Silva Martins; Silvia de Lima } \\
\text { Vieira; Wanessa Cassemiro } \\
\text { Fernandes; Claudinei Alves } \\
\text { Santana. }\end{array}$ & $\begin{array}{l}\text { Cuidados farmacêuticos na } \\
\text { adesão da terapia } \\
\text { medicamentosa oral em } \\
\text { pacientes onco-pediátricos. }\end{array}$ & $\begin{array}{l}\text { Esta pesquisa foi realizada através da } \\
\text { revisão de artigos científicos nas bases } \\
\text { de dados. Para isto, foram utilizadas as } \\
\text { palavras chaves: "adesão à medicação } \\
\text { oral", "câncer pediátrico", "orientação } \\
\text { farmacêutica" e seus equivalentes em } \\
\text { língua estrangeira (inglês), que } \\
\text { resultaram na listagem total de } 16 \\
\text { artigos. }\end{array}$ & $\begin{array}{l}\text { A adesão à terapia por via oral é uma das } \\
\text { formas de tratamento da doença, alguns } \\
\text { fatores podem influenciar a adesão, tais } \\
\text { como: o comportamento do doente, dados } \\
\text { sociodemográficos, como a idade, educação e } \\
\text { nível de conhecimento das crianças, } \\
\text { principalmente nas primeiras fases de vida. É } \\
\text { preciso orientação e manejo no cuidado ao } \\
\text { paciente onco-pediátrico. }\end{array}$ & $\begin{array}{l}\text { O acompanhamento, orientação e } \\
\text { monitoramento pelo profissional capacitado } \\
\text { são fundamentais para cuidado do câncer } \\
\text { pediátrico. }\end{array}$ \\
\hline $\begin{array}{l}\text { 11-Erica Assis Alves, Gabriel } \\
\text { Guimarães Tavares1, Leonardo } \\
\text { Luiz Borges. }\end{array}$ & $\begin{array}{l}\text { Importância da atenção } \\
\text { farmacêutica para a } \\
\text { quimioterapia antitumoral. }\end{array}$ & $\begin{array}{l}\text { Foi realizada uma revisão bibliográfica } \\
\text { nas principais plataformas de bases, } \\
\text { selecionando publicações de } 2009 \text { a } \\
2019 \text {. }\end{array}$ & $\begin{array}{l}\text { Dos resultados obtidos foram selecionados } 32 \\
\text { artigos, } 2 \text { teses de doutorado, } 2 \text { dissertações } \\
\text { de mestrado e } 3 \text { trabalhos de conclusão de } \\
\text { curso. }\end{array}$ & $\begin{array}{l}\text { Podemos concluir que o farmacêutico } \\
\text { atuante da área de oncologia deve ser um } \\
\text { profissional comprometido em cuidar de } \\
\text { pacientes debilitados, fragilizados física e } \\
\text { psicologicamente, também deve saber } \\
\text { orientar tanto os pacientes, quanto seus } \\
\text { familiares, utilizando palavras sensatas e de } \\
\text { conforto. }\end{array}$ \\
\hline $\begin{array}{l}\text { 12-Karina da Silva Aguiar; Jamile } \\
\text { Machado dos Santos; Mônica } \\
\text { Cristina Cambrussi; }\end{array}$ & $\begin{array}{l}\text { Segurança do paciente e o } \\
\text { valor da intervenção } \\
\text { farmacêutica em um hospital } \\
\text { oncológico. }\end{array}$ & $\begin{array}{l}\text { Estudo observacional e retrospectivo } \\
\text { realizado em um hospital oncológico. } \\
\text { De julho a agosto de } 2016 \text {, onde foram } \\
\text { avaliadas por farmacêuticos prescrições } \\
\text { contendo antineoplásicos e fármacos } \\
\text { adjuvantes ao tratamento. Os problemas } \\
\text { detectados relacionados a } \\
\text { medicamentos foram classificados e } \\
\text { analisados quanto ao medicamento, à } \\
\text { intervenção farmacêutica, à } \\
\text { aceitabilidade e à caracterização do } \\
\text { erro. }\end{array}$ & $\begin{array}{l}\text { Foram avaliadas } 6.104 \text { prescrições e } 12.128 \\
\text { medicamentos. Identificaram-se problemas } \\
\text { relacionados a medicamentos em } 274(4,5 \%) \\
\text { prescrições. Quando reunidos, os problemas } \\
\text { envolvendo dose representaram } 32,1 \%(\mathrm{n}=98) \\
\text { do total. Em } 13 \text { casos }(13,3 \%) \text { a variação da } \\
\text { dose prescrita em relação à correta foi maior } \\
\text { do que } 50 \% \text {. As principais intervenções } \\
\text { foram inclusão de informações }(\mathrm{n}=117 \text {; } \\
36,1 \%) \text { e alteração de dose }(\mathrm{n}=97 ; 29,9 \%) \text {. }\end{array}$ & $\begin{array}{l}\text { Ações simples de serem implantadas, como } \\
\text { análise de prescrições, são capazes de } \\
\text { identificar e prevenir problemas } \\
\text { relacionados a medicamentos, evitar perdas } \\
\text { financeiras e agregar imensurável valor na } \\
\text { segurança do paciente. }\end{array}$ \\
\hline $\begin{array}{l}\text { 13-Laís de Souza Gonçalves } \\
\text { Eugênio; Osni Lázaro Pinheiro. }\end{array}$ & $\begin{array}{l}\text { Paciente oncológico em } \\
\text { tratamento medicamentoso: } \\
\text { Subsídios para implantação } \\
\text { de um programa de atenção } \\
\text { Farmacêutica. }\end{array}$ & $\begin{array}{l}\text { Trata-se de estudo observacional e } \\
\text { transversal } \\
\text { ambulatoriais. }\end{array}$ & $\begin{array}{l}\text { Verificou-se a adesão ao tratamento com a } \\
\text { Escala de Morisky e os hábitos dos pacientes } \\
\text { com questionário validado por juízes. } \\
\text { Participaram } 52 \text { pacientes, } 52 \% \text { com alta e } \\
48 \% \text { com falhas na adesão. Relataram que }\end{array}$ & $\begin{array}{l}\text { Verificou-se a adesão ao tratamento com a } \\
\text { Escala de Morisky e os hábitos dos } \\
\text { pacientes com questionário validado por } \\
\text { juízes. Participaram } 52 \text { pacientes, } 52 \% \text { com } \\
\text { alta e } 48 \% \text { com falhas na adesão. Relataram }\end{array}$ \\
\hline
\end{tabular}




\begin{tabular}{|c|c|c|c|c|}
\hline & & & $\begin{array}{l}\text { dúvidas foram esclarecidas (63\%) e não } \\
\text { conheciam efeitos dos medicamentos }(65 \%) \text {, } \\
\text { sendo apontados efeitos indesejáveis }(64 \%) \text { e } \\
\text { necessidade de maiores informações }(58 \%) \text {, } \\
\text { por meio de contato. }\end{array}$ & $\begin{array}{l}\text { que dúvidas foram esclarecidas (63\%) e não } \\
\text { conheciam efeitos dos medicamentos } \\
(65 \%) \text {, sendo apontados efeitos indesejáveis } \\
(64 \%) \text { e necessidade de maiores } \\
\text { informações }(58 \%) \text {, por meio de contato. }\end{array}$ \\
\hline $\begin{array}{l}\text { 14-Priscila Helena Marietto } \\
\text { Figueira; Renata Rosa Veloso } \\
\text { Cataldo; Elaine Silva Miranda; } \\
\text { Gabriela Bittencourt Gonza-lez } \\
\text { Mosegui. }\end{array}$ & $\begin{array}{l}\text { Método estruturado para a } \\
\text { prática de conciliação } \\
\text { medicamentosa em hospital } \\
\text { /oncológico. }\end{array}$ & $\begin{array}{l}\text { Foi realizado um estudo exploratório, } \\
\text { em um hospital oncológico de grande } \\
\text { porte no Rio de Janeiro, aplicando-se o } \\
\text { método na internação de pacientes nos } \\
\text { Serviços de Ginecologia e Oncologia } \\
\text { do hospital. O método foi aplicado a } \\
201 \text { pacientes internados, permitindo a } \\
\text { mensuração da frequência da } \\
\text { conciliação medicamentosa instituída. }\end{array}$ & $\begin{array}{l}\text { Do total de pacientes entrevistados, } 89,6 \% \\
\text { eram do sexo feminino; } 28,2 \% \text { tinham entre } \\
61 \text { a } 70 \text { anos e } 25,2 \% \text { tinham entre } 51 \text { a } 60 \\
\text { anos. O principal motivo de internação foi o } \\
\text { procedimento cirúrgico }(69,3 \%) \text {. Por meio do } \\
\text { método proposto, foram identificadas } \\
\text { discrepâncias; essas foram registradas de } \\
\text { acordo com a classificação de Otero. }\end{array}$ & $\begin{array}{l}\text { A partir do método proposto, os } \\
\text { medicamentos utilizados anteriormente à } \\
\text { internação podem ser comparados com a } \\
\text { prescrição médica de internação e as } \\
\text { discrepâncias analisadas, aumentando a } \\
\text { qualidade do cuidado na rotina hospitalar }\end{array}$ \\
\hline $\begin{array}{l}\text { 15-Lívia Christina Almeida da } \\
\text { Silva; Poliana Oliveira Lemos de } \\
\text { Brito; Cristiane Dominice Melo; } \\
\text { Angela Falcai; Ione Cristina de } \\
\text { Paiva Pereira. }\end{array}$ & $\begin{array}{l}\text { Contribuições da atenção } \\
\text { farmacêtica a pacientes em } \\
\text { tratamento Oncológico. }\end{array}$ & $\begin{array}{l}\text { Trata-se de uma pesquisa bibliográfica } \\
\text { descritiva com o intuito de identificar } \\
\text { publicações a respeito das contribuições } \\
\text { da Atenção Farmacêutica ao paciente } \\
\text { oncológico em tratamento, baseada em } \\
\text { pesquisas realizadas no período de } 2007 \\
\text { a } 2017 \text {, utilizando-se artigos e teses } \\
\text { relacionados ao tema proposto. Para } \\
\text { localizar publicações será realizada uma } \\
\text { busca nas bases de dados online BVS, } \\
\text { SciELO, Google acadêmico e Pubmed } \\
\text { nos meses de setembro de } 2016 \text { a abril } \\
\text { de } 2017 \text {. }\end{array}$ & $\begin{array}{l}\text { Desta forma, conclui-se que a participação } \\
\text { ativa do farmacêutico junto aos pacientes em } \\
\text { tratamento e junto à equipe multiprofissional } \\
\text { se faz necessária, pois este profissional possui } \\
\text { qualificações para desempenhar na oncologia } \\
\text { papel administrativo e clínico cooperando } \\
\text { para uma terapia segura aos pacientes em } \\
\text { tratamento e também com os membros da } \\
\text { equipe. }\end{array}$ & $\begin{array}{l}\text { Baseado no levantamento de dados dos } \\
\text { artigos selecionados observou-se que a } \\
\text { prática da atenção farmacêutica é uma } \\
\text { atividade promissora por inúmeras razões. }\end{array}$ \\
\hline
\end{tabular}

Fonte: Autoras (2021). 


\section{Conclusão}

Com base no levantamento de dados, observou-se que a prática do cuidado farmacêutico é necessária e promissora por inúmeras razões. Pode-se, através deste trabalho mostrar a importância do profissional farmacêutico no cuidado ao paciente oncológico.

Dentre as atribuições, que podemos citar como importantes no cuidado farmacêutico ao paciente, tem-se: avaliação da prescrição, conciliação medicamentosa, orientação sobre uso de medicações e manejo das reações adversas; assim como, utilização de medicações de suporte durante o tratamento quimioterápico e dispensação. Com isso, podemos mostrar a competência que é disponibilizada à classe farmacêutica.

Avaliando a proposição desta revisão integrativa frente aos resultados encontrados nos artigos incluídos, evidencia-se que apesar de já existirem muitos trabalhos sobre cuidados farmacêuticos na literatura, ainda são poucos os estabelecimentos de saúde que prestam adequadamente esse tipo de serviço ou que o possuem implantado. Portanto a necessidade em trabalhos futuros de verificar as instituições que já possuem seus instrumentos de cuidado farmacêuticos em uso.

\section{Referências}

Aguiar, K. S., Santos, J. M., Cambrussi1, M. C., Carneiro, S. P. \& Carneiro, M. B. (2018). Segurança do paciente e o valor da intervenção farmacêutica em um hospital oncológico. Einstein, 16(1), 1-7.

Alberti, F. F., Cardoso, M. B. S., Canterle, L. P. \& Donini, E. K. (2018). Cuidado farmacêutico aplicado a mulheres com câncer de mama na Atenção Primária à Saúde. Revista Saúde, 44(1), 1-8.

Almeida, R. O., Braga, D. A. O., Souza, M. A., Barros, K. B. N. T. \& Vasconcelos, L. M. O. (2017). Implantação do cuidado farmacêutico em pacientes oncológicos em um núcleo de apoio à saúde da família. http://publicacoesacademicas.unicatolicaquixada.edu.br/index.p hp/mostracientificafarmacia/article/view/1798

Alves, E. A., Tavares, G. G. \& Borges, L. L. (2020). Importância da atenção farmacêutica para a quimioterapia antitumoral. Revista Brasileira Militar de Ciências. 6 (15), 8-17.

Calado, D. S., Tavares, D. H. C. \& Bezerra, G. C. (2019). O papel da atenção farmacêutica na redução das reações adversas associados ao tratamento de pacientes oncológicos. Rev. Bra. Edu. Saúde, 9(3), 94-99.

Chan, A., Shih, V. Chiang J, et al. (2013). Clinical pharmacy services and research for lymphoma patients at a cancer center. Journal of Oncology Pharmacy Practice, 19(1), 24-30.

Estrela, C. (2018). Metodologia Científica: Ciência, Ensino, Pesquisa. Editora Artes Médicas. 5.2) Pereira A. S. et al. (2018). Metodologia da pesquisa científica. UFSM.

Eugênio, L. S. G. \& Pinheiro, O. L. (2018). Paciente oncológico em tratamento medicamentoso: subsídios para implantação de um programa de atenção farmacêutica. Temas em Saúde, 18(2), 216-237.

Ferracini, F. T. \& Filho, W. B. M. (2012). Farmácia Clínica, segurança na prática hospitalar. Atheneu.

Figueira, P. H. M., Miranda, E. S. \& Mosegui, G. B. M. (2019). Método estruturado para a prática de conciliação medicamentosa em hospital oncológico. Revista Eletrônica Sistemas \& Gestão. 14(3), 1-8.

Leão, A. M., Dias, J. P. \& S, P. K. (2012). Atenção Farmacêutica no Tratamento Oncológico em uma Instituição Pública de Montes Claros. Rev Bras Farm Hosp Serv Saúde, 3(1), 11-14.

Lombardi, N. F. (2016). O serviço de Cuidado Farmacêutico na Atenção Primária à Saúde do Município de Curitiba PR. [Dissertação de Mestrado, Universidade Federal do Paraná].

Medeiros, J. A., Melo, A. P. F. M. \& Torres, V. M. (2019) Atuação do farmacêutico clínico hospitalar em pacientes oncológicos frente ao avanço na legislação brasileira. Rev. Bra. Edu. Saúde, 9(3), 56-65.

Pereira, L. R. L., Farias, M. R. F. \& Castro, M. S. (orgs) (2018). PROFARMA: Programa de atualização em ciências farmacêuticas: Ciclo 1, Porto Alegre: Artmed Panamericana.

Rangel, C. O., Gelatti, G. T., Salazar, R. F. S., Colet, C. F., Bandeira, V. A. C. \& Horn, R. C. (2020). Avaliação da adesão ao tratamento com Tamoxifeno por mulheres com câncer de mama. Rev. Epidemiol. Controle Infecç, 10(1), 86-93.

Rech, A. B. K., Francellino, M. A. M. \& Colacite, J. (2019) Atuação do farmacêutico na oncologia - uma revisão de literatura. Rev. Uningá, 56(4), 44-55. 
Research, Society and Development, v. 10, n. 5, e37410514987, 2021

(CC BY 4.0) | ISSN 2525-3409 | DOI: http://dx.doi.org/10.33448/rsd-v10i5.149887

Ribeiro M. A. S., Tuma, I. L., Nery, E. D. R. \& Marcos, J. F. (2009). Farmacêutico em oncologia: interfaces administrativas e clínicas. Pharmacia Brasileira: Conselho Federal de Farmácia. https://www.cff.org.br/sistemas/geral/revista/pdf/70/encarte_pb70.pdf

Sandna, L. F. S., Alves, H. H. S., Cinara, V. P, Saraiva, H. S. T. T. \& Barros, K. B. N. T. (2018). Evidências do cuidado farmacêutico na prática clínica da oncologia. Rev Fac Ciênc Méd, 20(2): 77-81.

Santos, J. P., Azevedo, R. M. H. S., Araújo, P. L., Bendicho, M. T. \& Xavier, R. M. F. X. (2020). Cuidado farmacêutico em UTI oncológica. Braz. J. Hea., 3(3), 5697-5704.

Silva, J. S., Rodrigues, F. C. \& Braga, R. R. (2020). Cuidado Farmacêutico a pacientes em tratamento com Herceptin® (Transtuzumabe): um estudo de caso. Infarma Ciências Farmacêuticas. 32(1), 23-29.

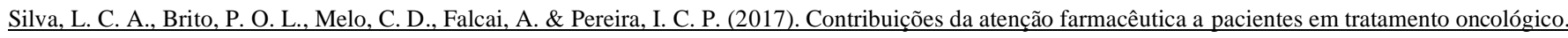
Rev. Investig, Bioméd., 9(2), 216-22.

Silva, L. C. et al. (2017). Contribuições da atenção farmacêutica a pacientes em tratamento oncológico. Revista de Investigação Biomédica, 9(2), 210 -217.

Silva, M. J. S. \& Osorio-de-Castro, C. G. S. (2019). Organização e práticas da assistência farmacêutica em oncologia no âmbito do Sistema Único de Saúde. Interface (Botucatu), 23, 1-17.

Simões, M. V. V., Martins, J. S., Vieira, S. L., Fernandes, W. C. \& Santana, C. A. (2020). Cuidados farmacêuticos na adesão da terapia medicamentosa oral em pacientes onco-pediátricos. Pubsaúde, 8, 1-8.

Storpitis, S. (2008). Farmácia Clínica e Atenção Farmacêutica. Guanabara.

WHO, World Health Organization. (2021). Health topics. Cancer. https://www.who.int/news-room/fact-sheets/detail/cancer. 